\title{
Isotermas de sorção de metais pesados em solos do cerrado de Goiás
}

\author{
$\overline{\text { Luiz F. C. } 0 \text { liveira }^{1} \text {, Mara L. Lemke-de-Castro }{ }^{2} \text {, Cristiane Rodrigues }{ }^{3} \& \text { Jácomo D. Borges }}{ }^{4}$
}

\section{RESUMO}

A contaminação do solo e das águas subterrâneas por metais pesados é, extremamente perniciosa por serem basntante persistentes no ambiente. O bjetivou-se, com este trabal ho, a análise da sorção do $\mathrm{Cu}, \mathrm{Cr}, \mathrm{Zn}, \mathrm{Cd}$, Pb e Ni em Latossolo Vermelho Acriférrico (LV wf), Argissolo Vermelho Eutrófico (PVe), Nitossolo Vermelho Eutroférrico (NVef) e N eossolo Q uartarênico (RQ) pelos modelos potencial e linear da isoterma Freundlich. Para o estabelecimento de isotermas de sorção adicionaram-se a 5,0 mL de solo e 50,0 mL de solução contendo o metal pesado a ser avaliado com diferentes concentrações, respeitandose os limites aceitáveis. As isotermas de sorção potencial e linear apresentaram bom ajuste para descrever o comportamento de adsorção dos metais pesados nas diferentes classes de solo estudadas. A sequência da retenção dos metais pesados em ordem decrescente, foi, para: 0 PVe: $\mathrm{Cr}^{+3}>\mathrm{Cr}^{+6}>\mathrm{Ni}^{+2}>\mathrm{Zn}^{+2}>\mathrm{Cu}^{+2}>\mathrm{Pb}^{+2}>\mathrm{Cd}^{+2} ; 0 \mathrm{LVwf} \mathrm{Cr}^{+3}>\mathrm{Cr}^{+6}>\mathrm{Ni}^{+2}$ $>\mathrm{Cu}^{+2}>\mathrm{Cd}^{+2}>\mathrm{Zn}^{+2}>\mathrm{Pb}^{+2} ; 0 \mathrm{RQ}: \mathrm{Cr}^{+6}>\mathrm{Cr}^{+3}>\mathrm{Cu}^{+2}>\mathrm{Pb}^{+2}>\mathrm{Ni}^{+2}>\mathrm{Zn}^{+2}>\mathrm{Cd}^{+2} \mathrm{e}$, para, $0 \mathrm{NVef:} \mathrm{Cr}^{+3}>\mathrm{Cr}^{+6}$ $>\mathrm{Pb}^{+2}>\mathrm{Cu}^{+2}>\mathrm{Zn}^{+2}>\mathrm{Ni}^{+2}>\mathrm{Cd}^{+2}$. $0 \mathrm{RQ}$ foi a classe de solo que apresentou menor retenção de metais em comparação com as demais classes de solo sendo, portanto, mais vulnerável à contaminação de águas subterrâneas.

Palavras-chave: Isoterma de Freundlich, fator de retardamento, adsorção de solutos, contaminação do solo

\section{Sorption isotherm of heavy metals in soils of 'Cerrado' of Goiás}

\begin{abstract}
The contamination of soil and groundwater by heavy metals is extremely damaging due to high persistence of heavy metals in the environment. This work targeted the analysis of the sorption in Oxisol Typic Acrustox(LVwf), Ultisol (PVe), Kandic Oxisol (N Vef) and Q uartzipsamment (RQ) of heavy metals $\mathrm{Cu}, \mathrm{Cr}, \mathrm{Zn}, \mathrm{Cd}, \mathrm{Pb}$ and $\mathrm{Ni}$. The sorption of the heavy metals in the soil was evaluated by batch method and described by potential and linear models of the Freundlich isotherm. For the establishment the of sorption isotherm, $50.0 \mathrm{~mL}$ of solution containing the heavy metal were added in $5.0 \mathrm{~mL}$ of soil, assessing with different concentrations within the acceptable soil limits. The potential and linear sorption isotherms presented good fitting to describe the behavior of adsorption of heavy metals in different classes of studied soil. The detention of heavy metals in, descending order was for PVe: $\mathrm{Cr}^{+3}>\mathrm{Cr}^{+6}>\mathrm{Ni}^{+2}>\mathrm{Zn}^{+2}>\mathrm{Cu}^{+2}>\mathrm{Pb}^{+2}>\mathrm{Cd}^{+2}$; for $\mathrm{LVwf}_{\mathrm{C}} \mathrm{Cr}^{+3}>$ $\mathrm{Cr}^{+6}>\mathrm{Ni}^{+2}>\mathrm{Cu}^{+2}>\mathrm{Cd}^{+2}>\mathrm{Zn}^{+2}>\mathrm{Pb}+2$; for $\mathrm{RQ}: \mathrm{Cr}^{+6}>\mathrm{Cr}^{+3}>\mathrm{Cu}^{+2}>\mathrm{Pb}^{+2}>\mathrm{Ni}^{+2}>\mathrm{Zn}^{+2}>\mathrm{Cd}^{+2}$ and for NVef: $\mathrm{Cr}^{+3}>\mathrm{Cr}^{+6}>\mathrm{Pb}^{+2}>\mathrm{Cu}^{+2}>\mathrm{Zn}^{+2}>\mathrm{Ni}^{+2}>\mathrm{Cd}^{+2}$. The Q uartzipsamment presented a less retention of metal compared to other classes of soil, and is therefore, more vulnerable to contamination of groundwater.
\end{abstract}

Key words: Freundlich isotherm, retardation factor, solute adsorption, soil pollution

\footnotetext{
1 Professor da UFLA, Bolsista em Produtividade do CN Pq, Departamento de Engenharia da UFLA, CP 3037, 37200-000, Lavras, MG. Fone: (35) 3829-1679, Email coutinho@deg.ufla.br

2 D outoranda em Agronomia, Solo e Água, Rodovia G oiânia/N ova Veneza, km 0, CEP 74001-970, Goiânia, G0. Fone: (62) 3521-1542, Email maralemke@uol.com.br ${ }_{3}^{3}$ Mestre em Agronomia, Solo e Água, Rodovia Goiânia/N ova Veneza, km 0, CEP 74001-970, Goiânia, G0. Fone (62) 3521-1542, Email crissolocia@yahoo.com.br 4 Professor da UFG, Rodovia Goiânia/N ova Veneza, km 0, 74001-970, Goiânia, G0. Fone (62) 3521-1542. Email jacomob@agro.ufg.br
} 


\section{INTRODUÇãO}

Designa-se metal pesado ao grupo de elementos que ocorrem em sistemas naturais em pequenas concentrações e apresentam densidade igual ou acima de $5000 \mathrm{~kg} \mathrm{~m}^{-3}$ (Reis et al., 2007). Em química ambiental esses elementos são também chamados elementos tóxicos, porém nem todo elemento tóxico é pesado, como é o caso dos ametais. Os íons $\mathrm{Cu}, \mathrm{Zn}, \mathrm{Fe}$, Mn e Ni, são micronutrientes enquadrados na lista dos metais pesados mas sua disponibilidade no solo e a concentração nas plantas e nos alimentos é o que limitam sua toxidez nas culturas agrícolas (Mattiazzo-Prezzoto, 1994).

Os metais pesados no solo, segundo Reis et al. (2007) e Tavares \& Carvalho (1992) resultam do intemperismo das rochas de origem, sobretudo aquelas ricas em sulfetos, óxidos, silicatos, fosfatos e carbonatos; contudo, teores mais elevados têm sido observados com frequência em diversas áreas em virtude das ações antrópicas, como sucessivas adições de fertilizantes, corretivos e defensivos que trazem metais pesados em suas formulações, rejeitos, escórias, lodos e uma infinidade de outros resíduos de natureza orgânica e inorgânica, associados ainda à deposição de efluentes e/ou atmosféricas oriundas de queima de combustíveis fósseis.

A contaminação do solo por metais pesados é extremamente perniciosa por serem altamente persistentes no ambiente. Ao contrário da maioria dos contaminantes orgânicos, os metais não podem ser degradados ou prontamente destoxificados pelos seres vivos tornando, assim, um agravante problema de poluição, ao longo do tempo. Muitos estudos nos quais se utilizaram resíduos industriais e urbanos como corretivo de solo, já foram realizados mas se verifica, na sua maioria, a toxicidade dos metais pesados em relação à produção agrícola, sua influência sobre o desenvolvimento das plantas e resíduo do metal no solo (Bissani et al., 2003; Luca et al., 1993).

Segundo Guilherme (1999) e Chang et al. (1994) os metais considerados importantes poluentes ambientais são: $\mathrm{As}, \mathrm{Bi}$, $\mathrm{Cd}, \mathrm{Cr}, \mathrm{Cu}, \mathrm{Hg}, \mathrm{Ni}, \mathrm{Sb}$ e $\mathrm{Zn}$, sendo os elementos $\mathrm{Cd}, \mathrm{Cu}, \mathrm{Zn}$ $\mathrm{e} \mathrm{Pb}$, os mais perigosos, devido à toxicidade e ao potencial de acumulação no solo.

O comportamento de alguns metais pesados em solos agricultáveis precisa ser mais estudado, sobretudo no que se refere à sua potencialidade quanto à retenção, lixiviação e contaminação, principalmente de águas subterrâneas possibilitando, desta forma, melhor recomendação na aplicação e disposição final desses produtos. O comportamento dos metais e a adequação do seu uso em solos de cultivo poderão revelar subsídios valiosos para amenizar o impacto ambiental negativo causado no solo e nas águas subterrâneas (Lima, 2004).

Para reduzir o risco potencial de contaminação é necessário se conhecer as interações entre os metais pesados e o solo, sua distribuição ao longo do perfil e sua disponibilidade à planta, pois esta prática inspira cuidados em razão da grande velocidade de decomposição da matéria orgânica em ambiente de clima tropical e pela capacidade do solo em adsorver os metais pesados (Corrêa et al., 2008).
A adsorção pode ser vista como um processo chave para se determinar o destino dos poluentes no sistema solo-água. Esta habilidade que o solo possui na retenção de solutos retarda seu transporte no perfil do solo. Características e propriedades como teor de argila e de matéria orgânica, capacidade de troca catiônica (CTC) e pH, entre outras, têm sido avaliadas como possíveis indicadores da capacidade de adsorção dos solutos pelo solo (Koskinen \& Harper, 1990).

A afinidade entre os cátions e as superfícies depende da carga elétrica, do raio hidratado e da configuração molecular do cátion. Em geral, cátions de maior valência são adsorvidos preferencialmente e cátions de menor raio tendem a substituir os de maior raio. No entanto, no caso de soluções com alta concentração de determinado cátion o mesmo pode substituir um cátion de maior preferência para a adsorção (La Grega et al., 1994).

O solo possui grande capacidade de retenção dos metais pesados, porém se esta capacidade for ultrapassada, os metais alterarão sua disponibilidade para o meio. Devido às alterações do $\mathrm{pH}$ o solo interage, alterando sua capacidade de troca catiônica e criando condições que facilitam a percolação. Desta forma, os metais pesados penetram na cadeia alimentar dos organismos vivos ou podem ser lixiviados, colocando em risco a qualidade dos sistemas aquáticos adjacentes e as águas subterrâneas (Pierangeli et al., 2004; 2007; Campos et al., 2007; Tavares \& Carvalho, 1992).

Quando o contaminante está associado à fase sólida, não é de conhecimento se este foi adsorvido à superfície do sólido, absorvido na estrutura do sólido, precipitado na superfície do sólido ou fracionado na matéria orgânica. Um termo genérico usado para descrever a partição de constituintes da fase líquida para a fase sólida e que não leva em consideração o mecanismo de retenção, é referido como sorção (Dias et al., 2001).

A sorção é, geralmente, quantificada pela função de distribuição que representa uma medida da partição do contaminante entre as fases sólida e líquida do sistema. A quantidade de soluto sorvida pelos sólidos é, em geral, uma função da sua concentração na solução (D'Agostinho \& Flues, 2006; Falone \& Vieira, 2004). As isotermas de sorção são equações matemáticas que descrevem as relações entre a quantidade de determinado elemento químico adsorvido e sua quantidade remanescente na solução de equilíbrio. As equações de Langmuir e Freundlich têm sido utilizadas com frequência para descrever a adsorção de diferentes elementos químicos pela fase coloidal do solo (Dias et al., 2001). Segundo Corrêa et al. (2008) e Mouta et al. (2008) a sorção de químicos no solo tem sido mais bem descrita pela isoterma de sorção de Freundlich, sendo a expressão mais comumente utilizada em problemas de contaminação de águas subterrâneas.

Em vista do exposto objetivou-se, neste trabalho, analisar o comportamento, no que refere à retenção de alguns metais pesados nas principais classes de solo agricultáveis do Estado de Goiás. Os resultados obtidos permitirão a avaliação da potencialidade de retenção, lixiviação e contaminação, principalmente de águas subterrâneas, pelos metais pesados para as classes de solos estudadas. 


\section{MATERIAL E MÉTODOS}

Devido à predominância nas áreas agricultáveis do Estado de Goiás foram selecionados, para o estudo de sorção de metais pesados, os seguintes solos: Latossolo Vermelho Acriférrico (LVwf) da região de Jataí; Argissolo Vermelho Eutrófico (PVe) da região de Goiânia, Nitossolo Vermelho Eutroférrico (NVef) da região de Ouro Verde e Neossolo Quartzarênico (RQ) da região de Caçú/Serranópolis. Para cada classe de solo foram feitas as amostragens do horizonte diagnóstico (horizonte B), devido ao fato de que, neste horizonte, ocorrem os maiores gradientes texturais e estruturais que afetam diretamente os mecanismos de sorção e movimento de água os quais estão diretamente associados ao transporte de solutos no solo. Para tal se coletaram nos horizontes diagnósticos, amostras deformadas e indeformadas, em triplicata.

Os pontos de amostragem foram previamente selecionados com base na ocorrência das classes de solo estudadas na presença da atividade agrícola. Para as análises químicas, granulométrica e massa específica de partículas se empregaram as amostras deformadas, as quais foram retiradas com auxílio de um trado, enquanto as amostras indeformadas foram utilizadas na determinação da massa específica do solo.

Realizaram-se as caracterizações físicas e químicas dos solos estudados nos laboratórios da Escola de Agronomia e Engenharia de Alimentos da Universidade Federal de Goiás, da empresa SoloCria e da Embrapa Arroz e Feijão, segundo as metodologias preconizadas pela EMBRAPA (1997). As análises mineralógicas da fração argila foram realizadas no difratômetro de raios- $X$ do Laboratório de Mecânica das Rochas da Divisão de Geotecnia de Furnas, localizado em Aparecida de Goiânia.

Determinaram-se, para a caracterização química, os teores de cátions do complexo sortivo e o $\mathrm{pH}$ em água e em $\mathrm{KCl}$. Como é a fração argila que torna o solo mais ou menos ativo, realizou-se o ensaio de ataque sulfúrico que decompõe somente os minerais secundários (argilominerais e óxidos de ferro e alumínio) que permitiram a obtenção dos índices de intemperismo $\mathrm{K}_{\mathrm{i}}$ e $\mathrm{K}_{\mathrm{r}}$ (Eq. 1 e 2) e a caracterização do grau de intemperização do solo com base na sua composição química; para tal, fez-se a determinação dos óxidos $\left(\mathrm{SiO}_{2}, \mathrm{Al}_{2} \mathrm{O}_{3}\right.$, $\mathrm{Fe}_{2} \mathrm{O}_{3}, \mathrm{TiO}_{2}$ e $\mathrm{P}_{2} \mathrm{O}_{5}$ ) extraídos pelo ataque sulfúrico (Embrapa, 1997)

$$
\begin{gathered}
\mathrm{K}_{\mathrm{i}}=\frac{1,7\left(\% \mathrm{SiO}_{2}\right)}{\% \mathrm{Al}_{2} \mathrm{O}_{3}} \\
\mathrm{~K}_{\mathrm{r}}=\frac{1,7\left(\% \mathrm{SiO}_{2}\right)}{\% \mathrm{Al}_{2} \mathrm{O}_{3}+0,64\left(\% \mathrm{Fe}_{2} \mathrm{O}_{3}\right)}
\end{gathered}
$$

Para os solos estudados, os metais pesados avaliados nos ensaios de sorção foram: cobre $(\mathrm{Cu})$, cromo $(\mathrm{Cr})$, zinco $(\mathrm{Zn})$, cádmio $(\mathrm{Cd})$, chumbo $(\mathrm{Pb})$ e níquel $(\mathrm{Ni})$. Inicialmente, as amostras indeformadas dos solos foram destorroadas e passadas em uma peneira de $2,0 \mathrm{~mm}$; posteriormente, elas foram secadas à sombra, por determinado período, até que a umidade apresentasse pequena variação obtendo-se, assim, as amostras de terra fina seca ao ar (TFSA).
A sorção dos solos foi avaliada pelo método da batelada. Para o estabelecimento de isotermas de sorção foram colocados, em um béquer, $5,0 \mathrm{~mL}$ de cada classe de solo em triplicata. Adicionaram-se, com auxílio de uma proveta, $50,0 \mathrm{~mL}$ da solução contendo os metais pesados cujas concentrações estão apresentadas na Tabela 1. As soluções foram preparadas em $\mathrm{CaCl}_{2} \cdot 2 \mathrm{H}_{2} \mathrm{O}\left(0,01 \mathrm{~mol} \mathrm{~L}^{-1}\right)$ como eletrólito-suporte empregando-se, como fonte dos metais, o sulfato de chumbo, sulfato de níquel, sulfato de zinco, sulfato de cobre, sulfato de cromo e cloreto de cádmio, respeitando-se as concentrações máximas aceitáveis em diferentes países (Chaves et al., 2008; Correia et al., 2007; Arantes et al., 2006).

Tabela 1. Reagentes e concentrações empregadas no ensaio de sorção

\begin{tabular}{lrrrrr}
\hline & \multicolumn{5}{c}{ Solução } \\
\cline { 2 - 6 } Metal & A & \multicolumn{5}{c}{ B } & \multicolumn{1}{c}{ D } & \multicolumn{1}{c}{ E } \\
\cline { 2 - 6 } & \multicolumn{5}{c}{ Concentração $\left(\mathbf{m g ~ L}^{-1}\right)$} \\
$\mathrm{Cr}^{+3}$ & 0,02 & 60,00 & 285,00 & 600,00 & 1700,00 \\
$\mathrm{Cr}^{+6}$ & 0,20 & 92,00 & 470,00 & 1350,00 & 2900,00 \\
$\mathrm{Zn}^{+2}$ & 0,40 & 94,80 & 515,00 & 2900,00 & 6450,00 \\
$\mathrm{Cu}^{+2}$ & 0,20 & 94,00 & 479,00 & 1800,00 & 3900,00 \\
$\mathrm{Ni}^{+2}$ & 0,01 & 10,50 & 97,80 & 179,00 & 390,00 \\
$\mathrm{~Pb}^{+2}$ & 0,10 & 92,70 & 483,00 & 1040,00 & 1380,00 \\
$\mathrm{Cd}^{+2}$ & 0,10 & 1,80 & 19,00 & 46,60 & 105,70 \\
\hline
\end{tabular}

Com a finalidade de se aumentar o contato da solução com o solo, os béqueres foram colocados em uma mesa agitadora e as suspensões agitadas durante $24 \mathrm{~h}$ (Mellis \& Rodella, 2008). Após a agitação, as amostras foram acondicionadas em uma caixa de isopor e mantidas na caixa durante $24 \mathrm{~h}$, para se obter a concentração de equilíbrio, conforme descrito por Correia et al. (2007); Arantes et al. (2006) e Jordão et al. (2000). A temperatura no interior da caixa foi monitorada com o auxílio de um termômetro para caixa de vacina.

Após o equilíbrio fez-se a filtragem do sobrenadante e as alíquotas foram transferidas para frascos de vidro âmbar e armazenadas em um frezer, até o momento da determinação da concentração do metal em solução pela técnica da espectrometria de absorção atômica. A concentração do metal adsorvido ao solo foi obtida pelo emprego da Eq. 3 .

$$
\mathrm{C}_{\mathrm{s}}=\left(\mathrm{C}_{\mathrm{i}}-\mathrm{C}_{\mathrm{f}}\right) \frac{\mathrm{Vol}}{\mathrm{m}}
$$

em que:

$\mathrm{C}_{\mathrm{s}}$ - concentração do metal adsorvido ao solo, $\mathrm{mg} \mathrm{kg}^{-1}$

$\mathrm{C}_{\mathrm{i}}$ e $\mathrm{C}_{\mathrm{f}}$ - concentração inicial e final da solução, $\mathrm{mg} \mathrm{L}^{-1}$

$\mathrm{m}$ - massa da TFSA adicionada ao Becker, $\mathrm{kg}$

Vol - volume da solução adicionada ao Becker, L

Para as diferentes classes de solo e metais pesados estudados, foram obtidas as concentrações adsorvidas para cada valor de $C_{i}$. De posse dessas informações se ajustaram as isotermas de sorção de Freundlich pelo modelo potencial (Eq. 4) e linear.

$$
\mathrm{C}_{\mathrm{s}}=\mathrm{K}_{\mathrm{f}} \mathrm{C}_{\mathrm{w}}^{\mathrm{N}}
$$

em que: 
$\mathrm{C}_{\mathrm{w}}$ - concentração do soluto em solução, $\mathrm{mg} \mathrm{L}^{-1}$;

$\mathrm{K}_{\mathrm{f}}$ e N - coeficiente e expoente da isoterma de Freundlich.

Quando o expoente $\mathrm{N}$ for unitário, ter-se-á uma isoterma linear que corresponde a um caso especial da isoterma de Freundlich. Neste caso, segundo Correia et al. (2007) e Arantes et al. (2006), o coeficiente de proporcionalidade da isoterma de Freundlich é chamado coeficiente de partição $\left(\mathrm{K}_{\mathrm{d}}\right)$.

De posse do coeficiente angular da isoterma de sorção linear $\left(\mathrm{K}_{\mathrm{d}}\right)$ determinou-se o respectivo fator de retardamento, pelo emprego da Eq. 5.

$$
\mathrm{R}=1+\mathrm{K}_{\mathrm{d}} \frac{\mathrm{D}_{\mathrm{s}}}{\mathrm{P}}
$$

em que:

$\mathrm{K}_{\mathrm{d}}$ - coeficiente de partição, $\mathrm{m}^{3} \mathrm{~kg}^{-1}$

$\mathrm{P}$ - porosidade total do solo, $\mathrm{m}^{3} \mathrm{~m}^{-3}$

$\mathrm{D}_{\mathrm{s}}$ - massa específica do solo, $\mathrm{kg} \mathrm{m}^{-3}$

Pelas análises das isotermas e dos fatores de retardamento, foi possível identificar as classes de solo que possuem predominância, no que se refere à adsorção dos metais pesados avaliados e, para cada classe de solo, a sequência da adsorção dos metais nos sítios de troca.

\section{RESULTADOS E DISCUSSÃO}

A Tabela 2 apresenta os resultados das análises físicas dos solos utilizados nos ensaios de sorção. Observou-se, para os PVe, LVwf e NVef, alta porcentagem da fração argila acima de $70 \%$, diferente do RQ que apresenta grande porcentagem da fração areia.

Tabela 2. Atributos físicos dos solos utilizados nos ensaios de sorção

\begin{tabular}{cccccccc}
\hline & \multicolumn{2}{c}{ Ds } & Dp & & Areia & Silte & Argila \\
\cline { 2 - 3 } \cline { 5 - 7 } Solo & \multicolumn{2}{c}{$\mathbf{k g ~ d m}^{-3}$} & & \multicolumn{3}{c}{$\%$} \\
PVe & 1,20 & 2,64 & & 12,7 & 11,2 & 76,1 \\
LVwf & 1,09 & 2,50 & & 11,8 & 17,8 & 70,4 \\
NVef & 1,10 & 2,65 & & 79,0 & 6,0 & 15,0 \\
RQ & 1,30 & 2,53 & & 7,5 & 13,6 & 78,9 \\
\hline
\end{tabular}

$\mathrm{D}_{\mathrm{s}}$ - massa específica do solo e $\mathrm{D}_{\mathrm{p}}$ - massa específica de partículas

Os índices $\mathrm{K}_{\mathrm{i}}$ e $\mathrm{K}_{\mathrm{r}}$ estão diretamente relacionados ao grau de alteração do solo sendo que, quanto maiores seus valores menor será o grau de intemperismo. $\mathrm{O} \mathrm{K}_{\mathrm{i}}$ fornece uma estimativa do grau de intemperismo de solos tropicais e subtropicais apresentando uma estimativa da relação caulinita e gibbsita nos solos. $\mathrm{O}$ índice $\mathrm{K}_{\mathrm{r}}$ é semelhante ao $\mathrm{K}_{\mathrm{i}}$, porém mais apropriado para indicar o grau de alteração dos minerais em solos em que a alta taxa de formação de óxidos de ferro contrasta com os baixos teores de alumina. Sugere-se, portanto, segundo a interpretação preconizada pela EMBRAPA (2006), que o PVe e o NVef são cauliníticos com boa retenção de cátions $(8,61<\mathrm{T}<15)$ e o LVwf e o RQ são solos gibbsíticos/oxídicos que possuem baixa capacidade de retenção de cátions $(1,61<\mathrm{T}<4,3)$ (Tabelas 3 e 4). Observa-
Tabela 3. Teor de Fe, Al, Si e Ti totais e índices $K_{i}$ e $K_{r}$ dos solos utilizados nos ensaios de sorção

\begin{tabular}{cccccccc}
\cline { 1 - 5 } Solo & $\mathrm{SiO}_{2}$ & $\mathrm{Al}_{2} \mathrm{O}_{3}$ & $\mathrm{Fe}_{2} \mathrm{O}_{3}$ & $\mathrm{TiO}_{2}$ & Óxidos* & \multirow{2}{*}{ Ki** } & \multirow{2}{*}{ Kr** } \\
\cline { 2 - 6 } & \multicolumn{5}{c}{$(\%)$} \\
PVe & 14,60 & 16,00 & 13,20 & 1,05 & 44,85 & 1,55 & 1,02 \\
LVwf & 9,02 & 22,50 & 25,50 & 1,60 & 58,62 & 0,68 & 0,40 \\
RQ & 9,50 & 21,70 & 22,00 & 1,40 & 54,60 & 0,74 & 0,45 \\
NVef & 24,40 & 24,00 & 18,00 & 1,40 & 67,80 & 1,73 & 1,17 \\
\hline
\end{tabular}

* óxidos - somatório de $\mathrm{SiO}_{2}, \mathrm{Al}_{2} \mathrm{O}_{3}, \mathrm{Fe}_{2} \mathrm{O}_{3}$ e $\mathrm{TiO}_{2}$; ** $-\mathrm{K}_{\mathrm{i}}$ e $\mathrm{K}_{2}$ ver equação 1 e 2

Tabela 4. Atributos químicos dos solos utilizados nos ensaios de sorção de metais

\begin{tabular}{|c|c|c|c|c|c|c|c|}
\hline \multirow{2}{*}{ Solo } & $S$ & $T$ & \multirow{2}{*}{ V (\%) } & \multicolumn{2}{|c|}{$\mathrm{pH}$} & \multirow{2}{*}{$\Delta \mathrm{pH}$} & \multirow{2}{*}{$\begin{array}{c}\mathrm{C} .0 \\
\left(\mathrm{~g} \mathrm{~kg}^{-1}\right.\end{array}$} \\
\hline & \multicolumn{2}{|c|}{$\left(\mathrm{cmolc} \mathrm{dm}^{-3}\right)$} & & $\mathrm{H}_{2} \mathrm{O}$ & $\mathrm{KCl}$ & & \\
\hline PVe & 8,53 & 11,43 & 74,64 & 6,60 & 5,00 & $-1,60$ & 4,10 \\
\hline LVwf & 0,35 & 3,65 & 9,59 & 3,80 & 5,00 & $+1,20$ & 7,00 \\
\hline $\mathrm{RQ}$ & 1,44 & 3,43 & 41,96 & 6,54 & 4,67 & $-1,87$ & 0,82 \\
\hline NVef & 7,27 & 9,27 & 78,43 & 6,00 & 5,70 & $-0,30$ & 4,00 \\
\hline
\end{tabular}

$\mathrm{S}$ - soma de bases, $\mathrm{T}$ - capacidade de troca catiônica, $\mathrm{V}$ - saturação por bases, C.O - carbono orgânico

se, pela Tabela 4, que no pH em água o PVe, RQ e o NVef apresentam acidez fraca a neutra mas o LVwf possui acidez elevada, com predominância de cargas positivas $(\Delta \mathrm{pH}$ positivo).

Pela análise dos difratogramas de raios-X, a mineralogia das argilas do PVe e do NVef têm, como principal mineral secundário, a caulinita, seguida de gibbsita e hematita no NVef e de ilita e magnetita no PVe. Observou-se para o LVwf uma mineralogia predominantemente oxídica. $\mathrm{O}$ resultado da análise dos difratogramas para os PVe, NVef e LVwf concorda com os valores obtidos para os índices $\mathrm{K}_{\mathrm{i}}$ e $\mathrm{K}_{\mathrm{r}}$; para o RQ não foi realizado a análise mineralógica no difratômetro de raios-X, em virtude do baixo porcentual da fração argila encontrado neste solo e por apresentar valores de $\mathrm{K}_{\mathrm{i}}$ e $\mathrm{K}_{\mathrm{r}} \leq 0,75$, o que confere, a este solo, predominância oxídica.

A Tabela 5 apresenta o resumo do ensaio de sorção dos metais pesados avaliados para as diferentes classes de solo, ou seja, os parâmetros ajustados para as isotermas de Freundlich potencial e linear e o fator de retardamento. De modo geral, as isotermas potenciais apresentaram melhor ajuste quando comparadas com a linear, com coeficientes de determinação $\left(\mathrm{R}^{2}\right)$ acima 0,95 . Este comportamento das isotermas ajustadas pode ser visualizado na Figura 1, em que estão

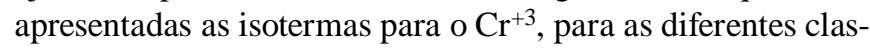
ses de solo.

De modo geral, o RQ possui menor capacidade de retenção dos metais avaliados (menores valores de $\mathrm{K}_{\mathrm{d}}$ e R); associado à movimentação de água nesta classe de solo, há maior risco de contaminação das águas subterrâneas. A baixa retenção dos metais neste solo está associada à baixa porcentagem da fração argila, responsável pelos sítios de troca (baixa CTC) e pelo tipo de argilomineral encontrado (predomínio de óxidos), concordando com Souza et al. (2006); Dias et al. 
(2003) e Sodré et al. (2001). Por outro lado, nos solos com maiores concentrações de argila, principalmente da caulinita (PVe e NVef), observou-se maior propensão, no que se refere à sorção dos metais no solo. Este comportamento fica mais evidente para o $\mathrm{Cr}^{+3}$ e $\mathrm{Cr}^{+6}$ em razão da maior competição desses elementos com os demais, que são todos bivalentes.

Analisando-se a capacidade de retenção dos metais pesados pelo fator de retardamento, tem-se, em ordem decrescente, para o PVe: $\mathrm{Cr}^{+3}>\mathrm{Cr}^{+6}>\mathrm{Ni}^{+2}>\mathrm{Zn}^{+2}>\mathrm{Cu}^{+2}>$ $\mathrm{Pb}^{+2}>\mathrm{Cd}^{+2}$; para o LVwf: $\mathrm{Cr}^{+3}>\mathrm{Cr}^{+6}>\mathrm{Ni}^{+2}>\mathrm{Cu}^{+2}>$ $\mathrm{Cd}^{+2}>\mathrm{Zn}^{+2}>\mathrm{Pb}^{+2}$; para o RQ: $\mathrm{Cr}^{+6}>\mathrm{Cr}^{+3}>\mathrm{Cu}^{+2}>\mathrm{Pb}^{+2}$ $>\mathrm{Ni}^{+2}>\mathrm{Zn}^{+2}>\mathrm{Cd}^{+2}$ e, para o NVef: $\mathrm{Cr}^{+3}>\mathrm{Cr}^{+6}>\mathrm{Pb}^{+2}>$ $\mathrm{Cu}^{+2}>\mathrm{Zn}^{+2}>\mathrm{Ni}^{+2}>\mathrm{Cd}^{+2}$. Segundo Pierangeli et al. (2007) o menor poder competitivo de $\mathrm{Cd}$, evidenciado pela redução da sua capacidade máxima de adsorção na presença dos outros metais, torna este elemento altamente pre-

Tabela 5. Isotermas de sorção linear e potencial e fator de retardamento para os metais pesados, nas diferentes classes de

\begin{tabular}{|c|c|c|c|c|c|c|}
\hline \multirow{2}{*}{ Solo } & \multicolumn{2}{|c|}{ Linear } & \multicolumn{3}{|c|}{ Potencial } & \multirow{2}{*}{$\mathbf{R}$} \\
\hline & Kd & $\mathbf{R}^{2 * *}$ & Kf & N & $\mathbf{R}^{2} * *$ & \\
\hline & \multicolumn{6}{|c|}{$\mathrm{Cr}^{+3}(25 \pm 2 \stackrel{\circ}{ } \mathrm{C})$} \\
\hline PVe & 3,4254 & 0,9439 & 6,0208 & 0,9487 & 0,9923 & 8,47 \\
\hline LVwf & 4,3848 & 0,9624 & 7,0744 & 0,9684 & 0,9964 & 9,53 \\
\hline $\mathrm{RQ}$ & 2,0837 & 0,9992 & 3,3857 & 0,9088 & 0,9950 & 6,95 \\
\hline \multirow[t]{2}{*}{ NVef } & 3,8573 & 0,9750 & 5,8220 & 0,9661 & 0,9962 & 11,23 \\
\hline & \multicolumn{6}{|c|}{$\mathrm{Cr}^{+6}\left(26 \pm 2 \stackrel{\circ}{ }{ }^{\circ}\right)$} \\
\hline PVe & 2,9123 & 0,8301 & 5,1825 & 0,8415 & 0,9590 & 7,35 \\
\hline LVwf & 3,8948 & 0,8620 & 5,6160 & 0,8803 & 0,9769 & 8,58 \\
\hline $\mathrm{RQ}$ & 2,6104 & 0,8925 & 4,8956 & 0,8711 & 0,9850 & 6,98 \\
\hline \multirow[t]{2}{*}{ NVef } & 2,6551 & 0,8372 & 5,3930 & 0,8223 & 0,9615 & 8,04 \\
\hline & \multicolumn{6}{|c|}{$\mathrm{Cd}^{+2}\left(26 \pm 2^{\circ} \mathrm{O}\right)$} \\
\hline PVe & 0,7115 & 0,9031 & 2,3359 & 0,7605 & 0,9559 & 2,55 \\
\hline LVwf & 1,7831 & 0,9906 & 2,9897 & 0,8671 & 0,9706 & 4,47 \\
\hline $\mathrm{RQ}$ & 0,6368 & 0,9745 & 2,2345 & 0,7117 & 0,9345 & 2,46 \\
\hline \multirow[t]{2}{*}{ NVef } & 0,4304 & 0,8566 & 1,1587 & 0,5947 & 0,7603 & 2,14 \\
\hline & \multicolumn{6}{|c|}{$\mathrm{Pb}^{+2}(26,5 \pm 2,5 \stackrel{\circ}{ } \mathrm{C})$} \\
\hline PVe & 1,0264 & 0,6382 & 2,8108 & 0,8928 & 0,9663 & 3,24 \\
\hline LVwf & 1,6552 & 0,8666 & 2,9365 & 0,9334 & 0,9925 & 4,22 \\
\hline $\mathrm{RQ}$ & 1,2169 & 0,9099 & 3,0571 & 0,8983 & 0,9737 & 3,79 \\
\hline \multirow[t]{2}{*}{ NVef } & 1,2701 & 0,995 & 4,9947 & 0,8028 & 0,9985 & 4,37 \\
\hline & \multicolumn{6}{|c|}{$\mathrm{Ni}^{+2}(27,25 \pm 3,8 \stackrel{9}{ } \mathrm{C})$} \\
\hline PVe & 2,1489 & 0,9445 & 3,1915 & 0,8308 & 0,9737 & 5,69 \\
\hline LVwf & 2,5358 & 0,9982 & 4,8423 & 0,8898 & 0,9969 & 5,94 \\
\hline RQ & 1,0753 & 0,7037 & 3,6932 & 0,8123 & 0,9796 & 3,46 \\
\hline \multirow[t]{2}{*}{ NVef } & 0,5955 & 0,6906 & 2,1577 & 0,7768 & 0,9907 & 2,58 \\
\hline & \multicolumn{6}{|c|}{$\mathrm{Cu}^{+2}\left(23,0 \pm 2,0^{\circ} \mathrm{C}\right)$} \\
\hline PVe & 1,1821 & 0,8887 & 6,1203 & 0,7811 & 0,9643 & 3,58 \\
\hline LVwf & 2,2628 & 0,9647 & 5,9976 & 0,8851 & 0,9906 & 5,40 \\
\hline $\mathrm{RQ}$ & 1,3394 & 0,8698 & 6,4750 & 0,7723 & 0,9548 & 4,02 \\
\hline \multirow[t]{2}{*}{ NVef } & 1,1029 & 0,928 & 5,4589 & 0,7884 & 0,9767 & 3,93 \\
\hline & \multicolumn{6}{|c|}{$\mathrm{Zn}^{+2}(23,0 \pm 2,0 \stackrel{\circ}{ } \mathrm{C})$} \\
\hline PVe & 1,3804 & 0,9845 & 4,9727 & 0,8694 & 0,9964 & 4,01 \\
\hline LVwf & 1,7358 & 0,9451 & 6,7999 & 0,8732 & 0,9918 & 4,38 \\
\hline $\mathrm{RQ}$ & 1,0692 & 0,8632 & 6,8619 & 0,8036 & 0,9968 & 3,45 \\
\hline NVef & 0,7752 & 0,7364 & 3,7018 & 0,8486 & 0,9924 & 3,06 \\
\hline
\end{tabular}

** significativo a $1 \%$
A.

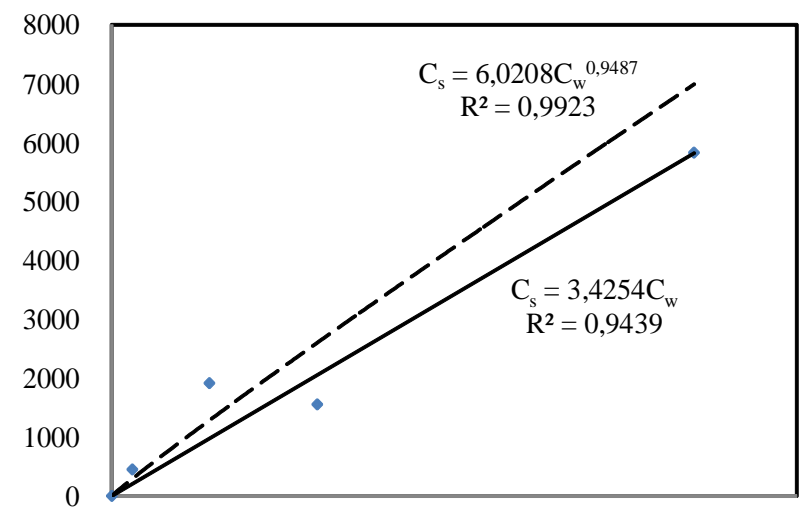

B.

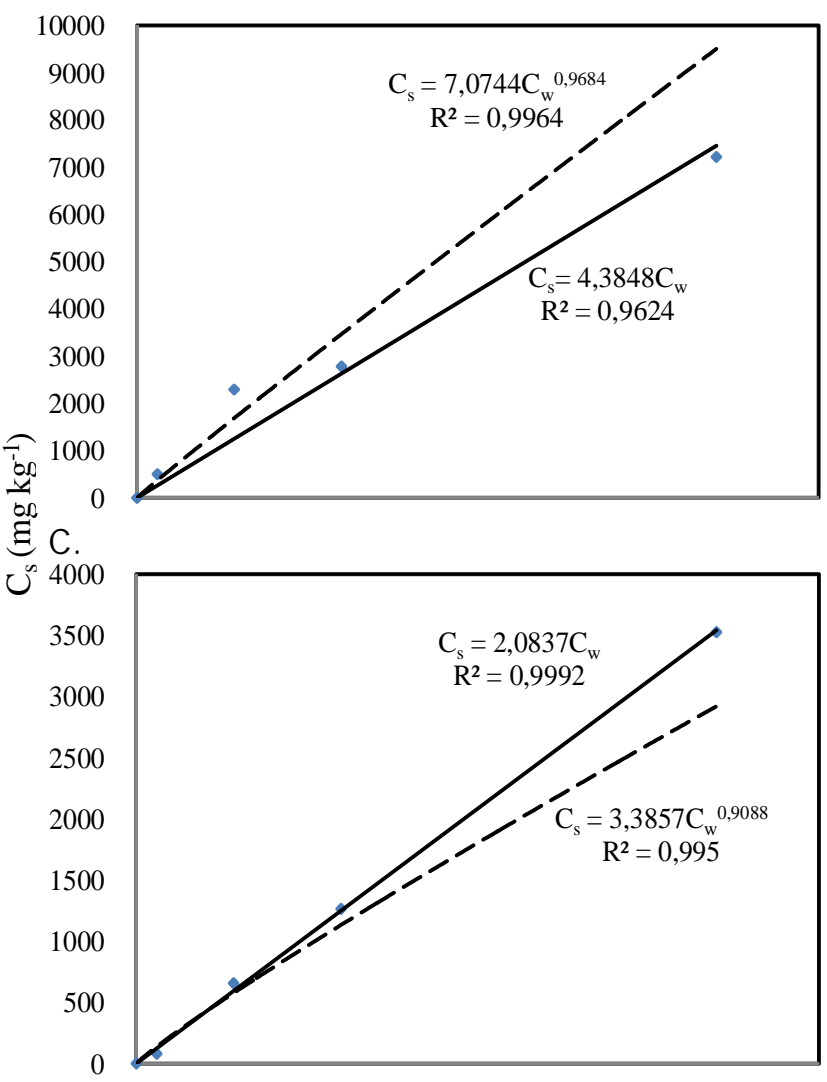

$\mathrm{D}$

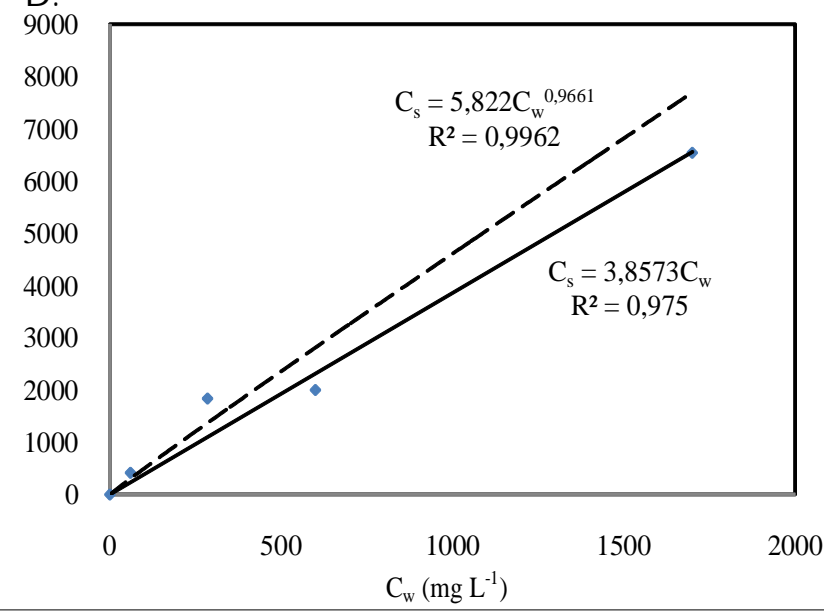

Figura 1. Isotermas de sorção potencial e linear para o $\mathrm{Cr}^{+3}$ para $\mathrm{PVe}$ (A); LVwf (B); RQ (C) e NVef (D) 
ocupante em solos tropicais mais intemperizados, como os Latossolos.

\section{CONCLUSÕES}

1. As isotermas de sorção ajustadas descrevem o comportamento de adsorção dos metais pesados estudados para as diferentes classes de solo agricultáveis, encontrados no cerrado do Estado de Goiás.

2. A sequência da retenção dos metais pesados estudados para as diferentes classes de solo, em ordem decrescente, foi: PVe: $\mathrm{Cr}^{+3}>\mathrm{Cr}^{+6}>\mathrm{Ni}^{+2}>\mathrm{Zn}^{+2}>\mathrm{Cu}^{+2}>\mathrm{Pb}^{+2}>\mathrm{Cd}^{+2}$; LVwf: $\mathrm{Cr}^{+3}>\mathrm{Cr}^{+6}>\mathrm{Ni}^{+2}>\mathrm{Cu}^{+2}>\mathrm{Cd}^{+2}>\mathrm{Zn}^{+2}>\mathrm{Pb}^{+2} ; \mathrm{RQ}: \mathrm{Cr}^{+6}>$ $\mathrm{Cr}^{+3}>\mathrm{Cu}^{+2}>\mathrm{Pb}^{+2}>\mathrm{Ni}^{+2}>\mathrm{Zn}^{+2}>\mathrm{Cd}^{+2}$ e, NVef: $\mathrm{Cr}^{+3}>\mathrm{Cr}^{+6}$ $>\mathrm{Pb}^{+2}>\mathrm{Cu}^{+2}>\mathrm{Zn}^{+2}>\mathrm{Ni}^{+2}>\mathrm{Cd}^{+2}$.

3. O RQ apresentou menores valores de $\mathrm{R}$ e, consequentemente, apresenta maior propensão à contaminação das águas subterrâneas; ter-se-ão, em seguida, o LVwf e, na sequência, os PVe e NVef.

\section{AGRADECIMENTOS}

Ao Conselho Nacional de Desenvolvimento Científico e Tecnológico $(\mathrm{CNPq})$, pelo aporte financeiro ao projeto de pesquisa "Retenção e transporte de metais pesados em solos agricultáveis do estado de Goiás”.

\section{LITERATURA CITADA}

Arantes, S. A. C. M.; Lima, J. M.; Nóbrega, J. C. A.; Guilherme, L. R. G.; Julião, L. G. F.; Jesus, E. A. Sorção de atrazina em solos representativos da sub-bacia do rio das Mortes, MG. Pesticidas: Revista de Ecotoxicologia e Meio Ambiente, v.16, p.101-110, 2006.

Bissani, C. A.; Camargo, F. A. O.; Ferreira, A. S.; Tedesco, M. J. Alterações de atributos químicos e biológicos de solo e rendimento de milho e soja pela utilização de resíduos de curtume e carbonífero. Revista Brasileira de Ciência do Solo, v.4, n.27, p.755-763, 2003.

Campos, M. L.; Guilherme, L. R. G.; Lopes, R. S.; Antunes, A. S.; Marques, J. J. G. S. M.; Curi, N. Teor e capacidade máxima de adsorção de arsênio em latossolos brasileiros. Revista Brasileira de Ciência do Solo, v.31, p.1311-1318, 2007.

Chang, A. C.; Page, A. L.; Warneke, J. E.; Grgureiv, E. Sequencial extraction of soil heavy metals following a sludge application. Jounal Environmental Quality, v.13, n.1, p.3338, 1994.

Chaves, L. H. G.; Souza, R. S.; Tito, G. A. Adsorção de zinco em argissolos do estado da Paraíba: efeito do pH. Revista Ciência Agronômica, v.39, n.4, p.511-516, 2008.

Correia, F. V.; Mercante, F. M.; Fabrício, A. C.; Campos, T. M. P.; Vargas Júnior, E.; Langenbach, T. Adsorção de atrazina em solo tropical sob plantio direto e convencional. Pesticidas: Revista de Ecotoxicologia e Meio Ambiente, v.17, p.37-46, 2007.
Corrêa, J. C.; Büll, L. T.; Paganini, W. S.; Guerrini, I. A. Disponibilidade de metais pesados em latossolo com aplicação superficial de escória, lama cal, lodos de esgoto e calcário. Pesquisa Agropecuária Brasileira, v.43, n.3, p.411-419, 2008.

D’Agostinho, A.; Flues, M. Determinação do coeficiente de distribuição (Kd) de benzo(a)pireno em solo por isotermas de sorção. Química Nova, v.29, n.4, p.657-661, 2006.

Dias, N. M. P.; Alleoni, L. R. F.; Casagrande, J. C.; Camargo, O. A. Isotermas de adsorção de cádmio em solos ácricos. Revista Brasileira de Engenharia Agrícola e Ambiental, v.5, n.2, p.229-234, 2001.

Dias, N. M. P.; Alleoni, L. R. F.; Casagrande, J. C.; Camargo, O. A. Energia livre da reação de adsorção de cádmio em latossolos ácricos. Ciência Rural, v.33, n.5, p.829-834, 2003.

EMBRAPA - Empresa Brasileira de Pesquisa Agropecuária. Centro Nacional de Pesquisa de Solos. Manual de métodos de análise de solos. Rio de Janeiro: EMBRAPA, 1997. 212p.

EMBRAPA - Empresa Brasileira de Pesquisa Agropecuária. Centro Nacional de Pesquisa de Solos. Sistema brasileiro de classificação de solos. Brasília: Embrapa. Produção de Informações; Rio de Janeiro: Embrapa Solos, 2006. $306 \mathrm{p}$.

Falone, S. Z.; Vieira, E. M. Adsorção/dessorção do explosivo tetril em turfa e em argissolo vermelho amarelo. Química Nova, v.27, n.6, p.849-854, 2004.

Guilherme, L. R. G. Poluição do solo e qualidade ambiental. In: Congresso Brasileiro de Ciência do Solo, 27. 1999. Brasília. Anais...Brasília: SBCS, 1999, CD-Rom.

Jordão, C. P.; Alves, N. M.; Pereira, J. L.; Bellato, C. R. Adsorção de íons $\mathrm{Cu}^{2+}$ em Latossolo Vermelho-Amarelo Húmico. Química Nova, v.23, n.1, p.5-11, 2000.

Koskinen, W. C.; Harper, S. S. The retention process: mechanisms. In: Cheng, H. H.; Bailey, G. W.; Green, R. E.; Spencer, W. F. Pesticides in the soil environment: processes, impacts, and modeling, Madison: Soil Science Society of America, 1990. p.51-78.

La Grega, M. D.; Buckingham, P. L.; Evans, J. C. Hazardous waste management. Singapore: McGraw-Hill, 1994. 1146p.

Lima, D. M. Sorção e deslocamento miscível da antrazina em amostras de latossolos. Lavras: UFLA, 2004. 66p. Dissertação Mestrado

Luca, S. J.; Ide, C. N.; Deus, A. B. S. The use of tannery sludges. Congresso da União International dos Químicos da Indústria do Couro, 22. Anais... Porto Alegre: AICSul, v.2, 1993, p.313-315.

Mattiazzo-Prezzoto, M. E. Comportamento de cobre, cádmio, crômio, níquel e zinco adicionados a solos de clima tropical em diferentes pH. Piracicaba: ESALQ: 1994. 197p. Errudição Livre Docência

Mellis, E. V.; Rodella, A. A. Influência do tempo de agitação na adsorção de $\mathrm{Cd}, \mathrm{Cu}$, Ni e $\mathrm{Zn}$ em latossolo tratado com lodo de esgoto. Bragantia, v.67, n.4, p.977-982, 2008.

Mouta, E. R.; Melo, W. J.; Soares, M. R.; Alleoni, L. R. F.; Casagrande, J. C. Adsorção de selênio em latossolos. Revista Brasileira de Ciência do Solo, n.32, p.1033-1041, 2008. 
Pierangeli, M. A. P.; Guilherme, L. R. G.; Curi, N.; Anderson, S. J.; Lima, J. M. Adsorção e dessorção de cádmio cobre e chumbo por amostras de latossolos pré-tratadas com fósforo. Revista Brasileira de Ciência do Solo, v.28, n.2, p.377-384, 2004.

Pierangeli, M. A. P.; Guilherme, L. R. G.; Curi, N.; Costa, E. T. S.; Lima, J. M.; Marques, J. J. G. S. M.; Figueiredo, L. F. P. Comportamento sortivo, individual e competitivo, de metais pesados em Latossolos com mineralogia contrastante. Revista Brasileira de Ciência do Solo, v.31, p.819-826, 2007.

Reis, T. H. P.; Guimarães, P. T. G.; Figueiredo, F. C.; Pozza, A. A. A.; Nogueira, F. D.; Rodrigues, C. R. O silício na nutrição e defesa de plantas. Belo Horizonte: EPAMIG. 2007, 12p. Boletim Técnico, 82.
Sodré, F. F.; Lenzi E.; Costa, A. C. S. Utilização de modelos físico-químicos de adsorção no estudo do comportamento do cobre em solos argilosos. Química Nova, v.24, n.3, p.324330, 2001.

Souza, R. S.; Chaves, L. H. G.; Fernandes, J. D. Adsorção de zinco e sua relação com características de solos do Estado da Paraíba. Revista Brasileira de Ciências Agrárias, v.1, n.único, p.1-6, 2006.

Tavares, T. M.; Carvalho, F. M. Avaliação da exposição de populações humanas a metais pesados no ambiente: Exemplos no Recôncavo Baiano. Química Nova, v.5, n.2, p.147-153, 1992. 